\title{
IncRNA KCNQ1OT1 knockdown inhibits colorectal cancer cell proliferation, migration and invasiveness via the PI3K/AKT pathway
}

\author{
QIAOBIN DUAN $^{1 *}$, LIANXU CAI $^{2 *}$, KEHONG ZHENG $^{2}$, CHUNHUI CUI $^{2}$, RENLI HUANG $^{2}$, \\ ZHENG ZHENG ${ }^{2}$, LANG XIE ${ }^{2}, \mathrm{CHENG}_{\mathrm{WU}}^{2}$, XIANG $\mathrm{YU}^{2}$ and JINLONG $\mathrm{YU}^{2}$ \\ ${ }^{1}$ Department of Hernia and Abdominal Surgery, The First People's Hospital of FoShan, \\ Affiliated FoShan Hospital of Sun Yat-sen University, Guangdong, Foshan 528000; ${ }^{2}$ Department of General Surgery, \\ Zhujiang Hospital, Southern Medical University, Guangdong, Guangzhou 510282, P.R. China
}

Received July 6, 2019; Accepted April 1, 2020

DOI: 10.3892/ol.2020.11619

\begin{abstract}
Colorectal cancer (CRC) is one of the most common primary malignancies worldwide. Numerous studies have demonstrated that long non-coding RNAs (lncRNAs) are considered as crucial regulators of tumor progression. In particular, upregulation of the lncRNA KCNQ1OT1 was reported in various types of malignancy as a promoter of tumor progression. However, the role and underlying mechanism of KCNQ1OT1 in CRC remain unclear. Thus, the present study aimed to investigate the role of KCNQ1OT1 in colorectal cancer through GEPIA, reverse transcription-quantitative PCR (RT-qPCR) and western blot analyses, and cell assays. GEPIA analysis demonstrated that high expression levels of KCNQ1OT1 in CRC tissues predicted a poor prognosis for patients with CRC. KCNQ1OT1 was overexpressed in CRC tissues and cell lines via RT-qPCR analysis. Furthermore, the results from the cell viability assay, colony formation assay, wound healing assay, invasion assay and flow cytometric analysis demonstrated that KCNQ1OT1 knockdown significantly inhibited CRC cell proliferation, migration and invasiveness, and promoted CRC cell apoptosis, leading to cell cycle arrest. Western blot analysis demonstrated that KCNQ1OT1 knockdown inhibited the PI3K/AKT signaling pathway. These results suggest that KCNQ1OT1 may act as an oncogene through the $\mathrm{PI} 3 \mathrm{~K} / \mathrm{AKT}$ signaling pathway in CRC.
\end{abstract}

Correspondence to: Professor Jinlong Yu, Department of General Surgery, Zhujiang Hospital, Southern Medical University, 253 Industrial Avenue, Guangdong, Guangzhou 510282, P.R. China E-mail: yujinlong640506@163.com

*Contributed equally

Key words: colorectal cancer, KCNQ1OT1, proliferation, migration, PI3K/AKT

\section{Introduction}

Colorectal cancer (CRC) is the third most diagnosed cancer worldwide with a high mortality rate, which reported 1.4 million new cases and 693,900 mortalities in 2012 (1). Based on the thorough understanding of the CRC pathological process, immunotherapy and targeted therapy have emerged and have greatly improved the prognosis of patients with CRC (2). However, because the occurrence and development of CRC is a complex process caused by numerous factors, the prognosis of patients with advanced-stage CRC remains unsatisfactory (3). It is therefore crucial to identify the underlying mechanisms of CRC tumorigenesis, in order to develop novel therapeutic strategies.

The human genome consists of $2 \%$ genes coding for proteins and several non-coding RNAs (ncRNAs) (4). Long non-coding (lnc)RNAs are a type of ncRNAs that are defined as transcripts with lengths $>200$ nucleotides, and that are not translated into proteins $(5,6)$. Although lncRNAs were previously considered as transcriptional noise, previous studies have reported that lncRNAs serve crucial roles in the regulation of biological processes involved in numerous diseases, including various types of cancer $(6,7)$. Previous studies have reported that the IncRNA KCNQ1OT1 is involved in the progression of multiple tumors, including tongue cancer, hepatocellular carcinoma, and breast cancer (8-10). However, the underlying mechanisms of KCNQ1OT1 in CRC development and metastasis remain unknown.

The present study investigated KCNQ1OT1 expression in CRC tissues and its association with the prognosis of patients with CRC. Furthermore, the role of KCNQ1OT1 in CRC cell proliferation, migratory and invasive abilities, apoptosis and the cell cycle was also analyzed. The underlying mechanisms of KCNQ1OT1 in CRC cells were subsequently investigated, which may help to provide a new therapeutic and prognostic method for colorectal cancer.

\section{Materials and methods}

Clinical samples. A total of 28 pairs of CRC and adjacent normal tissues $(>5 \mathrm{~cm}$ from the edge of tumor 
tissues) were collected from patients with CRC between June 2018 and January 2019 at Zhujiang Hospital, Southern Medical University, and preserved in liquid nitrogen. All tissue samples were confirmed by histopathology, and tumor staging was based on the NCCN Colon Cancer Guidelines (11). The clinicopathological information of all patients, including age, sex, tumor size, pathological differentiation and Tumor-Node-Metastasis TNM, were collected at the Department of General Surgery of Zhujiang Hospital, Southern Medical University. The patients recruited in the present study suffered from colon adenocarcinoma (COAD) or rectum adenocarcinoma (READ), and all data were analyzed together. The present study was approved by the Ethics Committee of Zhujiang Hospital. All patients provided written informed consent prior to the study.

Bioinformatics analysis. Gene Expression Profiling Interactive Analysis (GEPIA http://gepia.cancer-pku.cn/) is an online web server used for the analysis of RNA sequencing expression data of 9,736 tumor samples and 8,587 normal samples from The Cancer Genome Atlas (https://www.cancer.gov) and the Genotype-Tissue Expression (https://commonfund. nih.gov/GTEx) databases, using a standard processing pipeline (12). Following submission of an analysis request, GEPIA can provide the visual image results for users (12). The gene symbol KCNQ1OT1 was submitted on GEPIA for differential expression analysis between data from CRC and normal tissues, and provided results consisting of box plots, stage plots and survival analysis.

Cell culture. The human CRC cell lines RKO, SW620, colo320, HCT116 and LoVo, and the normal colon epithelial cell line NCM460 were obtained from the Southern Medical University Corporation. All cells were maintained in RPMI-1640 (HyClone; GE Healthcare Life Sciences) containing 10\% FBS (Serana Europe $\mathrm{GmbH}$ ) and placed at $37^{\circ} \mathrm{C}$ in a humidified incubator containing $5 \% \mathrm{CO}_{2}$.

PI3K inhibitor was purchased from Selleck Chemicals. The stably transfected SW620 and RKO cells were treated with $10 \mu \mathrm{M}$ PI3K inhibitor (LY294002) for $24 \mathrm{~h}$ (13) to suppress the PI3K/AKT signaling pathway when the density of cells reached $50 \%$.

$R N A$ extraction and reverse transcription-quantitative $(R T-q)$ $P C R$. Total RNA was extracted from the primary tissues and cell lines using TRIzol ${ }^{\mathrm{TM}}$ reagent (Takara Bio, Inc.), and reverse transcribed into cDNA with the PrimeScript RT Kit (Takara Bio, Inc.) according to the manufacturers' instructions. RT-qPCR reactions were performed as follows: $37^{\circ} \mathrm{C}$ for $15 \mathrm{~min}$, followed by $85^{\circ} \mathrm{C}$ for $5 \mathrm{sec}$. qPCR was performed using the TB Green premix (Takara Bio, Inc.) in a CFX96 real-time PCR detection system (Bio-Rad Laboratories, Inc.). The sequences of the primers (Sangon Biotech, Co., Ltd.) were as follows: KCNQ1OT1 forward, 5'-CAAGCAGCC AGAAGGATGAGAAGG-3' and reverse 5'-GGTCAGCAC CAGAAGGCAGAATG-3'; and GAPDH forward, 5'-TGC ACCACCAACTGCTTAGC-3' and reverse 5'-GGCATG CACTGTGGTCATGAG-3'. The relative expression level of KCNQ1OT1 normalized to endogenous control and expressed as $2^{-\Delta \Delta \mathrm{Cq}}(14)$.
Lentivirus production and transfection. Short hairpin RNAs (shRNAs) against human KCNQ1OT1 (sh-KCNQ1OT1-1 and sh-KCNQ1OT1-2) or scrambled oligonucleotides (sh-nc) were ligated into a LV-5 (EF-1 $\alpha \mathrm{F} / \mathrm{GFP}+\mathrm{Puro})$ vector (all from Shanghai GenePharm Co., Ltd.). Lentivirus solution $\left(10^{8} \mathrm{TU} / \mathrm{ml}\right)$ was transfected into the SW620 and RKO lines using Lipofectamine 3000 (Thermo Fisher Scientific, Inc,). The stably transfected cells were selected by puromycin selection $(5 \mu \mathrm{g} / \mathrm{ml})$ for $\geq 2$ weeks. Subsequent experiments were performed after 2 weeks of cell transfection.

Cell viability assay. The stably transfected SW620 and RKO cells were seeded into 96-well plates at the density of $2 \times 10^{3}$ cells per well. Cell viability was assessed every $24 \mathrm{~h}$ for 5 days using the Cell Counting-Kit 8 reagent (CCK-8; Beijing Transgen Biotech Co., Ltd.). Briefly, cells were incubated with $10 \mathrm{ul} \mathrm{CCK}-8$ solution for $2 \mathrm{~h}$ and absorbance was read at $450 \mathrm{~nm}$ on a microplate reader.

Colony formation assay. The stably transfected cells were seeded in 6-well plates at a density of $2 \times 10^{3}$ cells/well and cultured at $37^{\circ} \mathrm{C}$ for $12-14$ days. Once the colonies were visible, cells were washed twice with PBS, and colonies were fixed with $5 \%$ paraformaldehyde for $20 \mathrm{~min}$ at room temperature and stained with $0.1 \%$ crystal violet $(1 \mathrm{mg} / \mathrm{ml})$ for $20 \mathrm{~min}$. The stained colonies were counted, and the colony formation rate was calculated using ImageJ 8.0 software (National Institutes of Health).

Wound healing assay. The stably transfected cells were seeded into 6 -well plates at a density of $5 \times 10^{5}$ cells/well and cultured until they had reached $90 \%$ confluence. The complete medium was replaced with serum-free medium and the cell monolayer was scratched with a sterile $100-\mu 1$ pipette tip. After further culture for $48 \mathrm{~h}$, the wound gap was observed under an inverted light microscope (magnification $\mathrm{x} 100$ ). The extent of wound healing was calculated using the ImageJ 8.0 software. The wound healing percentage was calculated using the following formula: (Scratch healing area)/(original scratch area) $\mathrm{x} 100 \%$.

Invasion assays. The stably transfected cells were suspended in serum-free medium at a density of $2 \times 10^{5}$ cells $/ 200 \mu \mathrm{l}$ and seeded into the Matrigel-coated upper chamber $(8-\mu \mathrm{m}$ pore size) of Transwell inserts in 24 -well plates at $37^{\circ} \mathrm{C}$ for $1 \mathrm{~h}$. the lower chamber was filled with $600 \mu \mathrm{l}$ complete medium. Following $48 \mathrm{~h}$ of culture, cells remaining on the upper surface of the filter were swabbed off using cotton wool, and cells that had invaded the lower surface were fixed with $5 \%$ paraformaldehyde for $20 \mathrm{~min}$ at room temperature and stained with Giemsa for $10 \mathrm{~min}$. Cells were observed under an inverted light microscope (magnification $\mathrm{x} 200$ ), and the number of cells per field was calculated using ImageJ 8.0 software.

Cell cycle and apoptosis analysis. The stably transfected SW620 and RKO cells were trypsinized (without EDTA) and washed with PBS. For cell cycle analysis, $10^{5}$ cells $/ 100 \mu \mathrm{l}$ cells were fixed with $70 \%$ ethanol at $4^{\circ} \mathrm{C}$ for $12 \mathrm{~h}$ and resuspended in staining buffer containing $450 \mu \mathrm{l}$ propidium iodide (PI) and $50 \mu \mathrm{l}$ RNaseA in the dark for $30 \mathrm{~min}$ at room temperature 
A

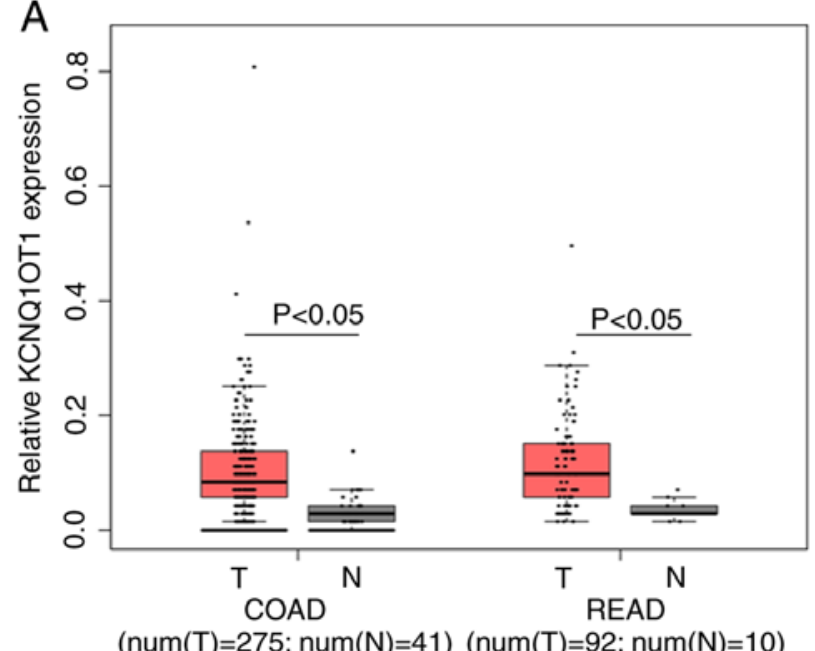

C

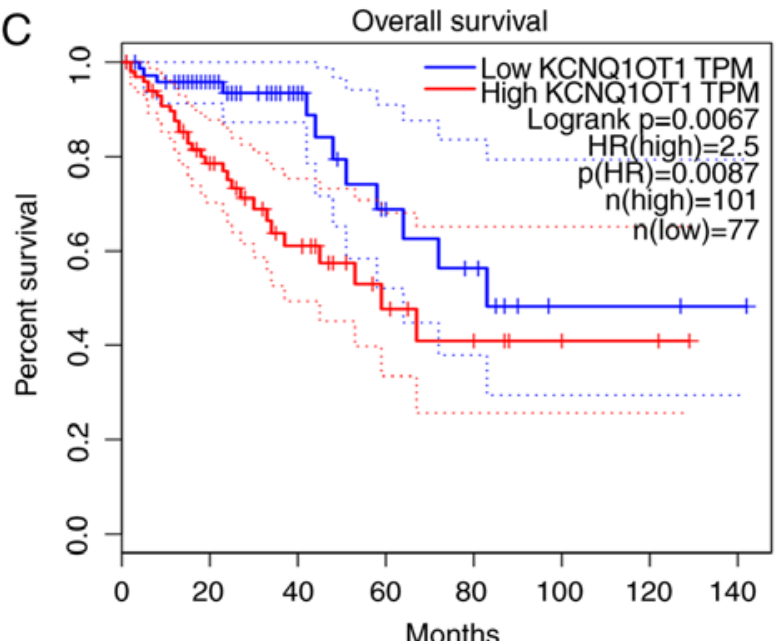

E

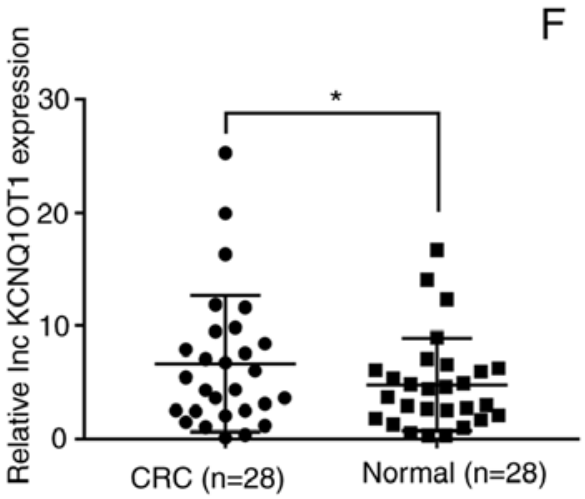

B

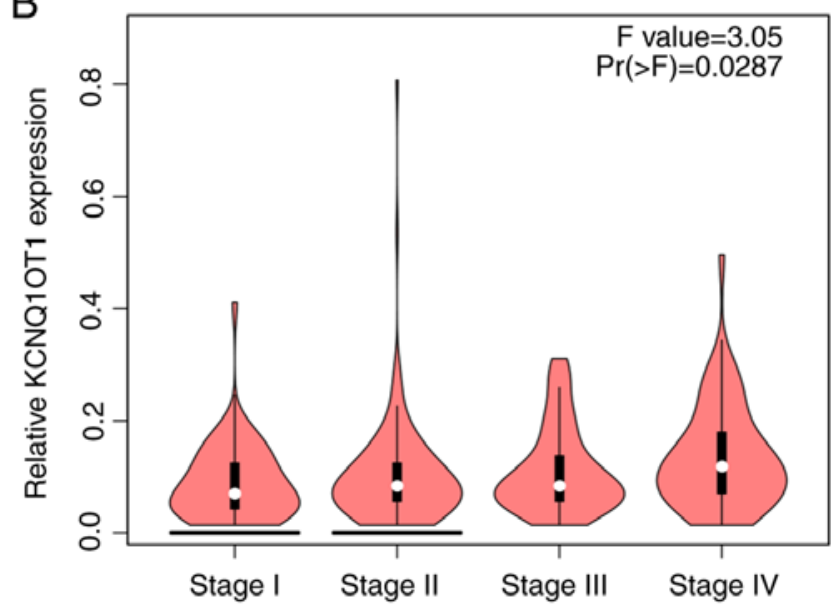

D

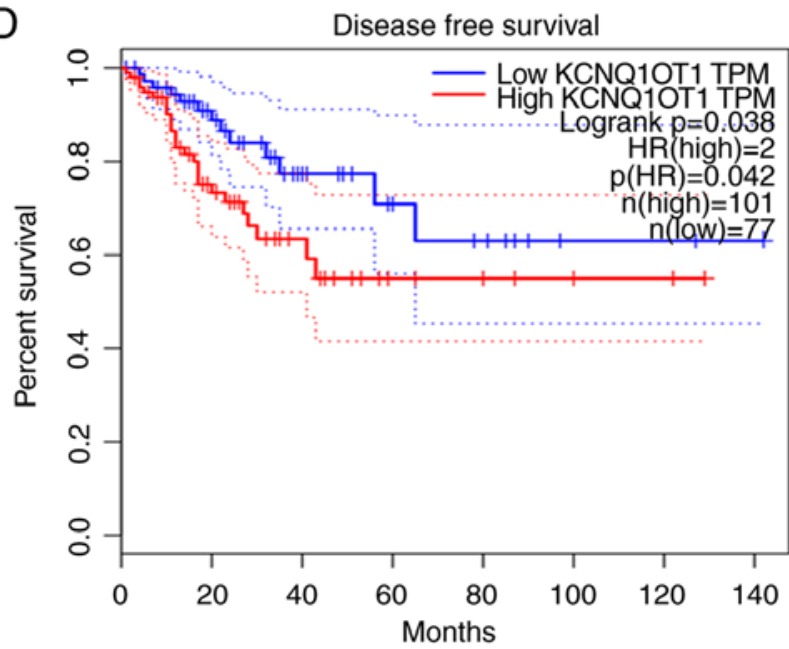

G

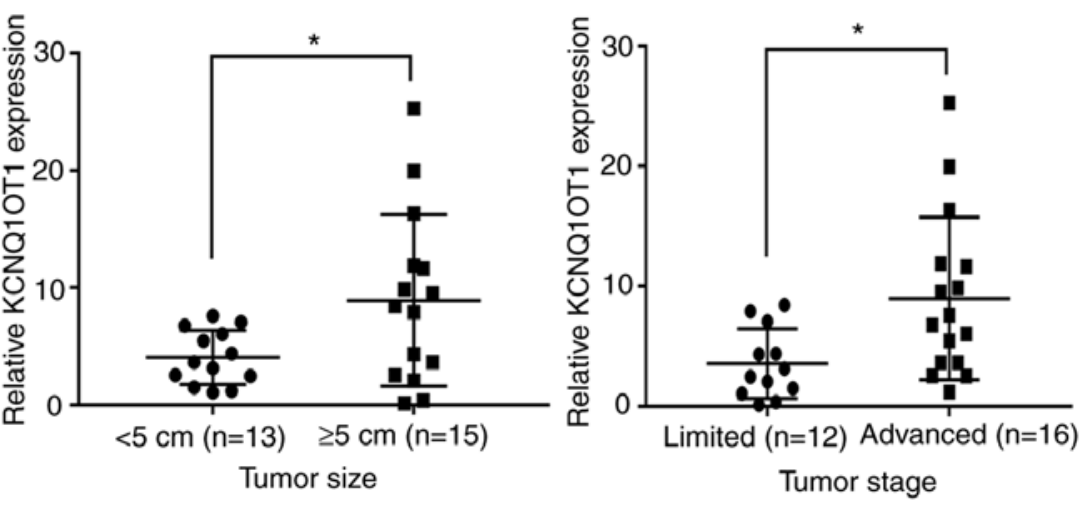

Figure 1. KCNQ1OT1 is upregulated in CRC tissues and associated with poor prognosis. (A) Relative expression of KCNQ1OT1 in CRC and normal tissues via GEPIA analysis (B) Relative expression of KCNQ1OT1 in CRC tissues from patients at different disease stages via GEPIA analysis. Association between KCNQ1OT1 expression and the (C) overall and (D) disease-free survival of patients with CRC patients via GEPIA analysis. (E) Relative expression of KCNQ1OT1 in 28 pairs of CRC and adjacent normal tissues (paired t-test). (F) Relative expression of KCNQ1OT1 in tumors $<5 \mathrm{~cm}$ ( $\mathrm{n}=13$ ) and $\geq 5 \mathrm{~cm}$ ( $\mathrm{n}=15$; unpaired $\mathrm{t}$-test). (G) Relative expression of KCNQ1OT1 in tumors from patients with limited stage $(\mathrm{n}=12)$ and advanced stage (n=16; unpaired t-test) disease. * $\mathrm{P}<0.05$. COAD, colon adenocarcinoma; CRC, colorectal cancer; GEPIA, Gene Expression Profiling Interactive Analysis; N, normal; READ, rectum adenocarcinoma; T, tumor.

(cat. no. KGA512; Nanjing KeyGen Biotech Co., Ltd.) For apoptosis analysis, the Annexin V APC/7AAD apoptosis kit (cat. no. KGF004; Nanjing KeyGen Biotech Co., Ltd.) was used to stain for early and late apoptotic cells according to the manufacturers' instructions. Subsequently, stained cells were analyzed using a BD FACS-Verse flow cytometer (BD Biosciences). Cell cycle distribution was obtained using
ModFit 3.2 software (Verity Software House, Inc.), and the apoptosis rate was calculated using FlowJo 7.6.1 software (FlowJo LLC).

Western blotting. Total proteins were extracted from cells and tissues using the Protein Extraction Kit (cat. no. KGP2100; Nanjing KeyGen Biotech Co., Ltd.) and quantified with a 

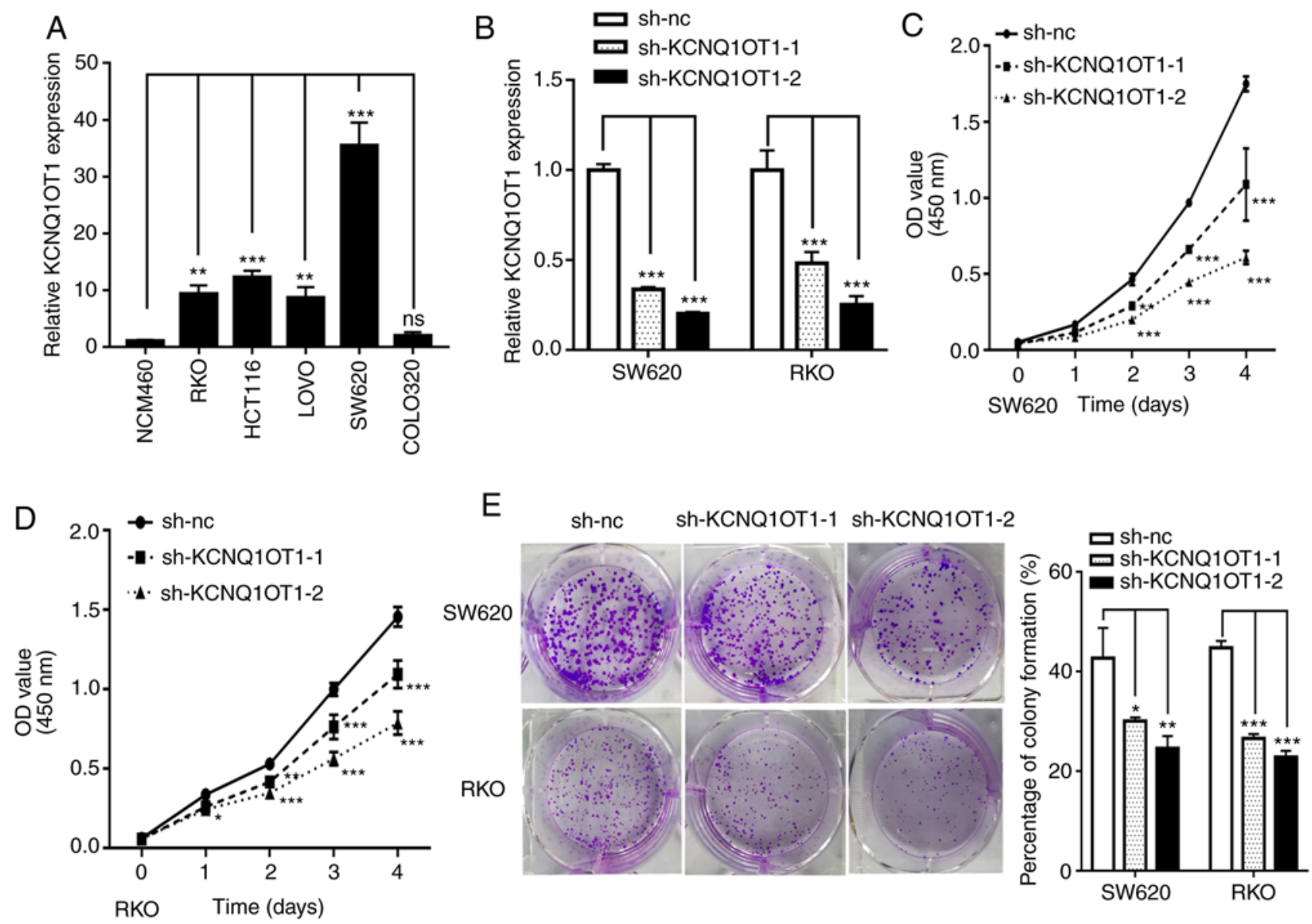

Figure 2. KCNQ1OT1 knockdown inhibits CRC cell proliferation in vitro. (A) Relative expression of KCNQ1OT1 in the CRC cell lines SW620, RKO, HCT116, LoVo and colo320, and the normal intestinal epithelial cell line NCM460. (B) Relative expression of KCNQ1OT1 in SW620 and RKO cells transfected with sh-nc, sh-KCNQ1OT1-1 and sh-KCNQ1OT1-2 constructs. Viability of (C) SW620 and (D) RKO cells following KCNQ1OT1 knockdown. (E) Colony forming capacity of SW620 and RKO cells following KCNQ1OT1 knockdown. ${ }^{*} \mathrm{P}<0.05,{ }^{* *} \mathrm{P}<0.01$ and ${ }^{* * *} \mathrm{P}<0.001$. CRC, colorectal cancer; nc, negative control; OD, optical density; Sh, short hairpin.

BCA assay (Nanjing KeyGen Biotech Co., Ltd. KGPBCA). Proteins (20 ug) were separated by 12\% SDS-PAGE (Fude Biological Technology, Co. Ltd.) and transferred onto polyvinylidene fluoride membranes (EMD Millipore). The membranes were blocked with 5\% skim milk (Fude Biological Technology, Co., Ltd.) for $1.5 \mathrm{~h}$ at room temperature, and incubated with primary antibodies against: PI3K (Wanleibio Co., Ltd.; cat. no. WL03380; 1:1,000), phosphorylated (p-) PI3K (Wanleibio Co., Ltd.; cat. no. WL0226; 1:1,000), AKT (Wanleibio Co., Ltd.; cat. no. WL003b; 1:1,000), p-AKT (Wanleibio Co., Ltd.; cat. no. WLP001a; 1:1,000), BAX (ProteinTech Group, Inc.; cat. no. 50599-2-lg; 1:5,000), BCL2 (ProteinTech Group, Inc.; cat. no. 12789-1-AP; 1:5,000) and GAPDH (Bioworld Technology, Inc.; cat. no. BS60630; $1: 10,000)$ overnight at $4^{\circ} \mathrm{C}$. The membranes were then incubated with goat-anti-rabbit secondary antibody (Fude Biological Technology, Co. Ltd.; cat. no. FDR007; 1:2,000) for $1 \mathrm{~h}$ at room temperature. The immuno-positive bands were visualized using ECL developer solution (Fude Biological Technology, Co., Ltd.) and an imaging system (Bio-Rad Laboratories, Inc.).

Statistical analysis. All statistical analyses were performed using GraphPad Prism 7.0 (GraphPad Software, Inc.). Data were presented as the means \pm standard deviation of at least three independent experiments. Differences between two groups were assessed using the t-test, whereas one-way ANOVA, followed by Newman-Keuls post-hoc test were used to compare differences between multiple groups $(>2)$. $\mathrm{P}<0.05$ was considered to indicate a statistically significant difference.

\section{Results}

KCNQ1OT1 expression is upregulated in CRC tissues and is associated with disease progression and poor survival in patients with CRC. Previous studies have reported that KCNQ1OT1 expression is upregulated in various types of tumor, including hepatocellular carcinoma, non-small cell lung cancer and tongue cancer $(8,9,15)$, and that this is correlated with poor prognosis in patients. In the present study, GEPIA was used to predict KCNQ1OT1 expression in CRC. The results from box plot analysis in GEPIA demonstrated that KCNQ1OT1 expression was significantly higher in tumor tissues compared with normal tissues in patients with COAD and READ $(\mathrm{P}<0.05$; Fig. 1A). The results from stage plot analysis in GEPIA demonstrated that KCNQ1OT1 expression increased with tumor stage 
A

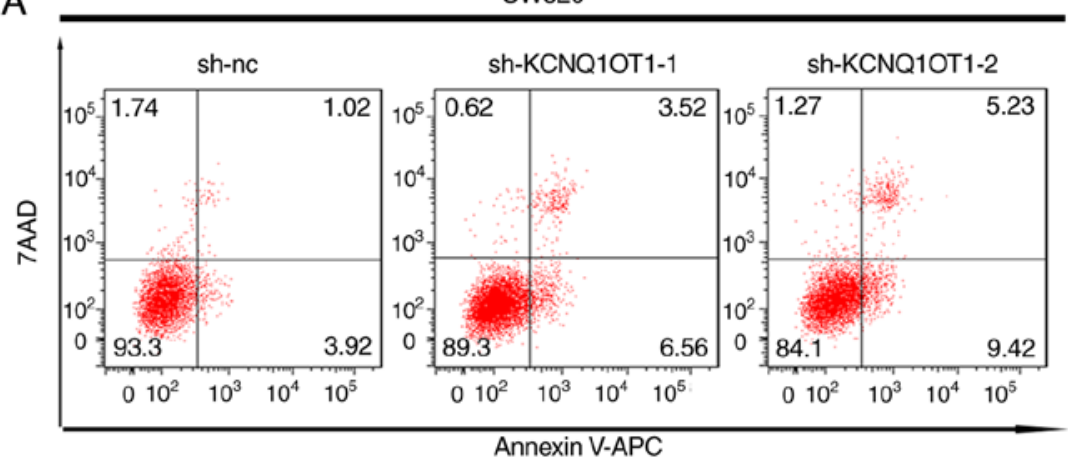

B

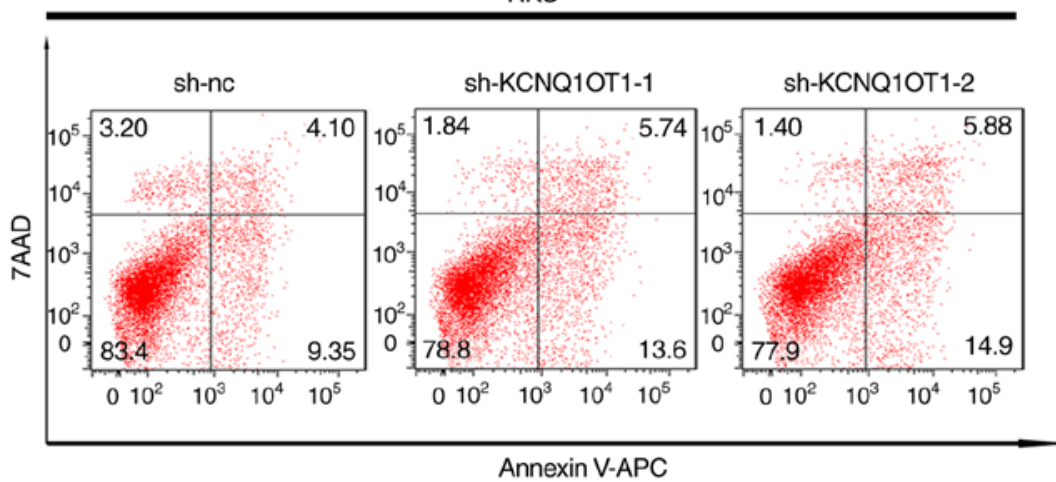

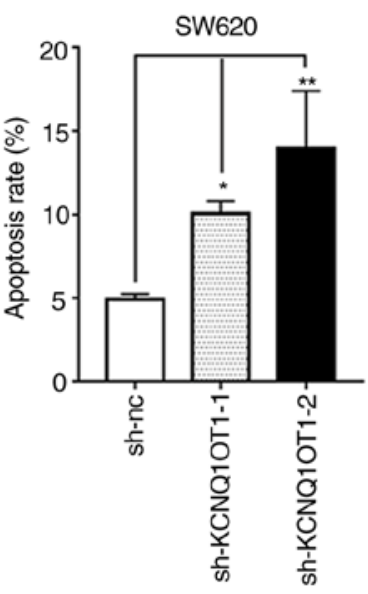

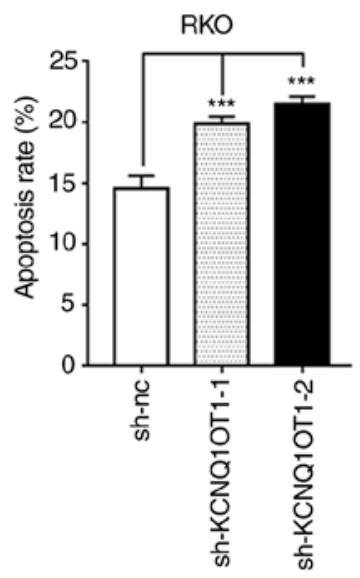

SW620
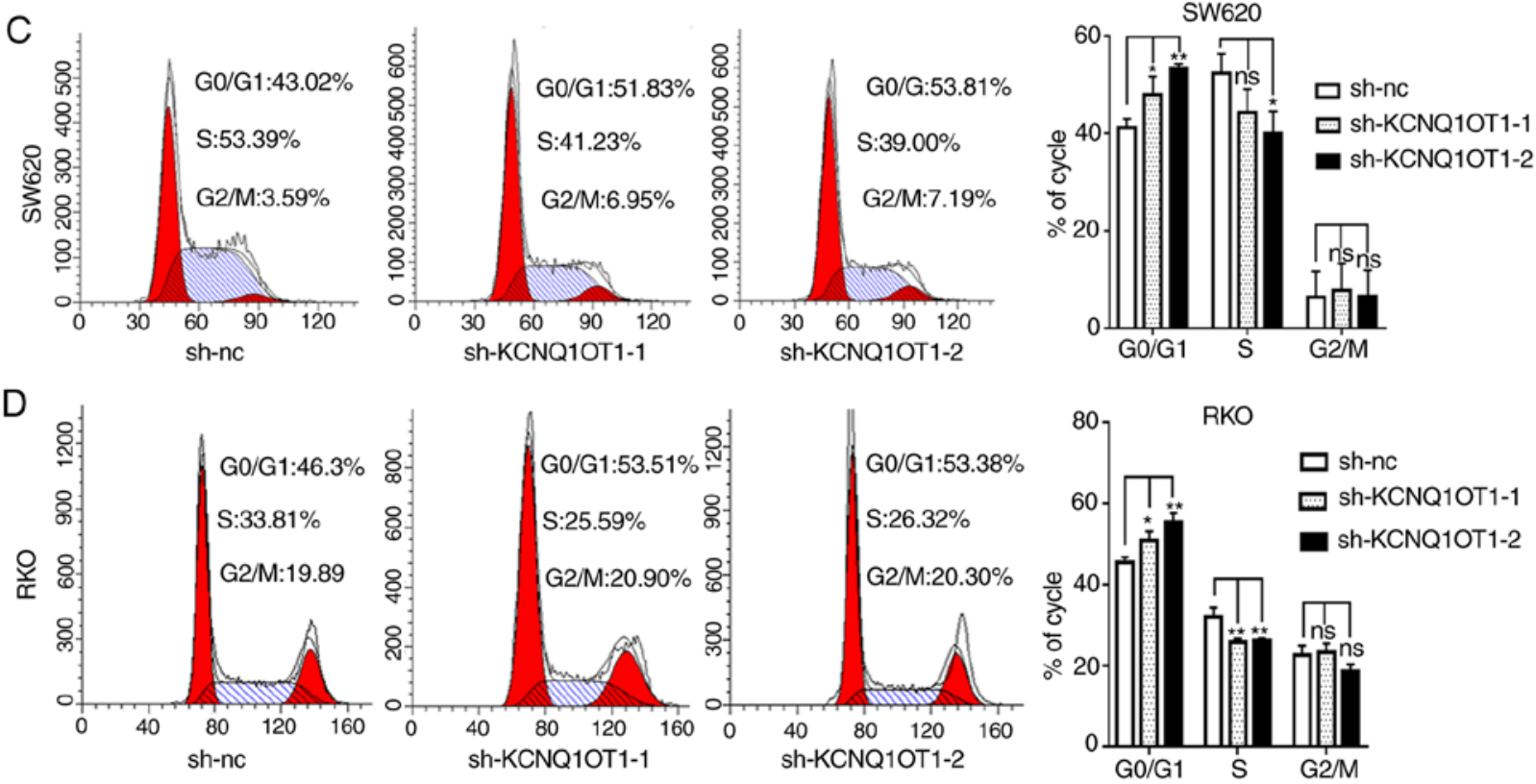

Figure 3. KCNQ1OT1 knockdown induces apoptosis and cell cycle arrest in colorectal cancer cells. Apoptosis rates in (A) SW620 and (B) RKO cells following KCNQ1OT1 knockdown. Cell cycle profiles of (C) SW620 and (D) RKO cells following KCNQ1OT1 knockdown. " P $<0.05,{ }^{* *} \mathrm{P}<0.01$ and ${ }^{* * * *} \mathrm{P}<0.001$. nc, negative control; ns, not significant; sh, short hairpin.

progression in patients with $\mathrm{CRC}(\mathrm{P}=0.0287$; Fig. 1B). Furthermore, results from survival analysis in GEPIA demonstrated that high KCNQ1OT1 expression in CRC tissues was associated with lower overall and disease-free survival times (Fig. 1C and D). These predicted results suggest that KCNQ1OT1 expression may be upregulated in
CRC tissues and may be associated with poor prognosis in patients with CRC.

Subsequently, 28 pairs of CRC and adjacent normal tissues were collected from patients with CRC, and KCNQ1OT1 expression was examined by RT-qPCR. The results demonstrated that the KCNQ1OT1 expression level was significantly 

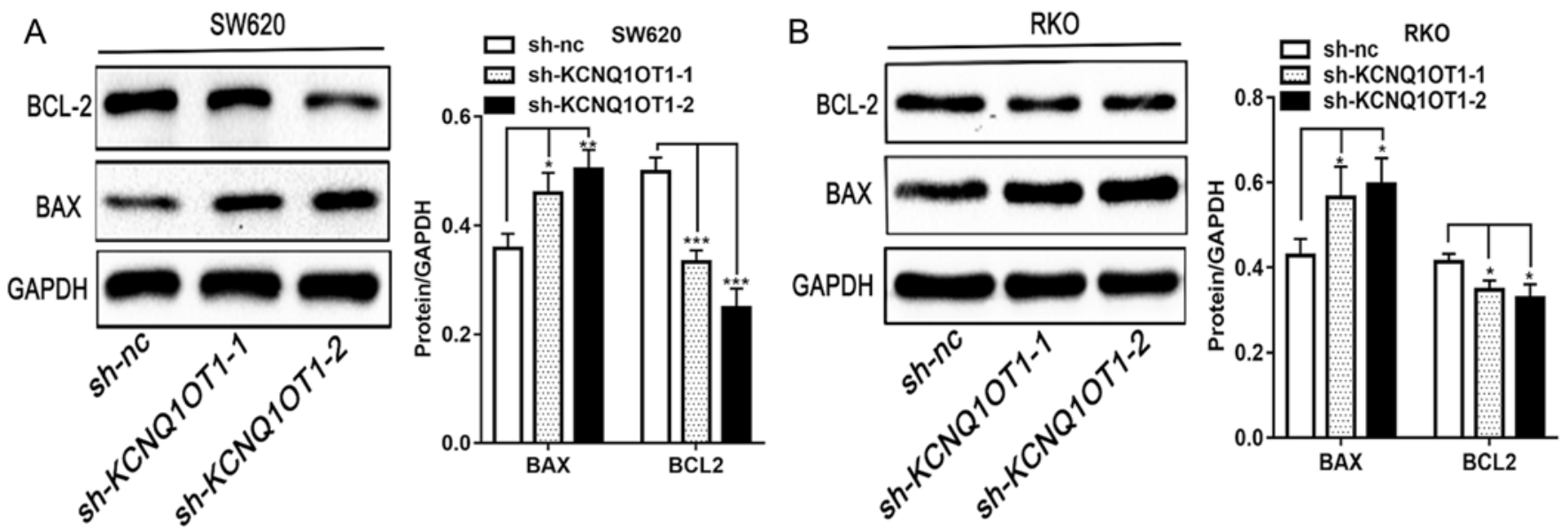

Figure 4. KCNQ1OT1 knockdown affects apoptotic protein expression in colorectal cancer cells. Relative expression of BAX and BCL2 proteins in KCNQ1OT1 knockdown (A) SW620 and (B) RKO cells. ${ }^{*} \mathrm{P}<0.05,{ }^{* *} \mathrm{P}<0.01$ and ${ }^{* * *} \mathrm{P}<0.001$. nc, negative control; Sh, short hairpin.

higher in CRC tissues compared with adjacent normal tissues $(\mathrm{P}=0.0142$; Fig 1E). Furthermore, KCNQ1OT1 expression level was significantly higher in CRC tissues obtained from large tumors $(\geq 5 \mathrm{~cm})$ compared with those obtained from small tumors $(<5 \mathrm{~cm} ; \mathrm{P}=0.0302 ;$ Fig. $1 \mathrm{~F})$. In addition, the KCNQ1OT1 expression level in CRC tissues obtained from patients with advanced stage CRC (IIIB+IIIC+IV) was significantly increased compared with those obtained from patients with limited stage disease (I+II+IIIA; P=0.0372; Fig. 1G). These findings indicate that KCNQ1OT1 may be associated with tumor growth and metastasis in CRC.

KCNQ1OT1 knockdown inhibits CRC cell proliferation in vitro. To investigate the role of KCNQ1OT1 in CRC growth, in vitro experiments were performed. Firstly, KCNQ1OT1 expression was assessed by RT-qPCR in five CRC cell lines and the normal NCM460 intestinal epithelial cell line. The results demonstrate that KCNQ1OT1 expression level was upregulated in four out of five CRC cell lines compared with the normal cell line (Fig. 2A). SW620 and RKO cells were subsequently selected to construct stably transfected cell lines using two KCNQ1OT1-specifc shRNAs lentivirus or negative control lentivirus. The results from RT-qPCR demonstrate that KCNQ1OT1 expression level was efficiently knocked down in the two cell lines following transfection (Fig. 2B). Subsequently, cell viability and colony formation assays were performed to investigate the effect of KCNQ1OT1 on CRC cell proliferation in vitro. The results demonstrate that KCNQ1OT1 knockdown significantly decreased SW620 and RKO cell viability (Fig. 2C and D). Furthermore, the results of the colony formation assay demonstrated that KCNQ1OT1 knockdown significantly inhibited clone formation capacity in SW620 and RKO cells (Fig. 2E). These findings confirm that KCNQ1OT1 knockdown inhibits CRC cell proliferation.

KCNQ1OT1 knockdown stimulates CRC cell apoptosis and cell cycle arrest. A previous study reported that abnormal cell cycle and apoptosis are associated with cancer growth (16). To better understand the underlying mechanism of KCNQ1OT1 in CRC cell proliferation, apoptosis and cell cycle distribution were evaluated in the present study. The results demonstrated that the apoptotic rates of KCNQ1OT1 knockdown SW620 and RKO cells were significantly increased compared with the control cells (Fig. 3A and B). Furthermore, as presented in Fig. 4A and B, the expression of the pro-apoptotic protein BAX and of the anti-apoptotic protein BCL-2, in KCNQ1OT1 knockdown CRC cells was significantly increased and decreased, respectively, compared with control group. The results from western blotting are therefore consistent with results from apoptosis detection by flow cytometry. In addition, the results from cell cycle analysis demonstrated that KCNQ1OT1 knockdown significantly increased the percentage of cells in the $G_{0} / G_{1}$ phase and decreased the percentage of cells in the $S$ phase in SW620 and RKO cell lines, compared with control cells (Fig. 3C and D). These findings suggest that downregulation of KCNQ1OT1 in CRC cells may induce apoptosis and arrest the cell cycle at the $G_{0} / G_{1}$ phase.

KCNQ1OT1 knockdown inhibits CRC cell migratory and invasive abilities. In order to investigate the biological function of KCNQ1OT1 in CRC cell invasiveness, a Transwell assay was performed. The results demonstrate that the invasive ability of KCNQ1OT1 knockdown cells was significantly decreased compared with control cells (Fig. 5A). Similarly, the results from the wound healing assay demonstrated that silencing KCNQ1OT1 in SW620 or RKO cells significantly decreased the scratch healing ability (Fig. 5B). These results indicate that KCNQ1OT1 knockdown may decrease CRC cell invasion in vitro.

KCNQ1OT1 knockdown inhibits the activation of the PI3K/AKT signaling pathway. Previous studies have reported that the PI3K/AKT pathway is one of the most commonly altered pathways in human cancer that is associated with the regulation of tumor growth and metastasis $(17,18)$. In order to identify the underlying mechanism of KCNQ1OT1 in CRC progression, the PI3K/AKT pathway was investigated. To do so, the expression of proteins from the PI3K/AKT pathway was analyzed by western blotting in CRC cells transfected with sh-KCNQ1OT1 or sh-nc. The results demonstrate that 

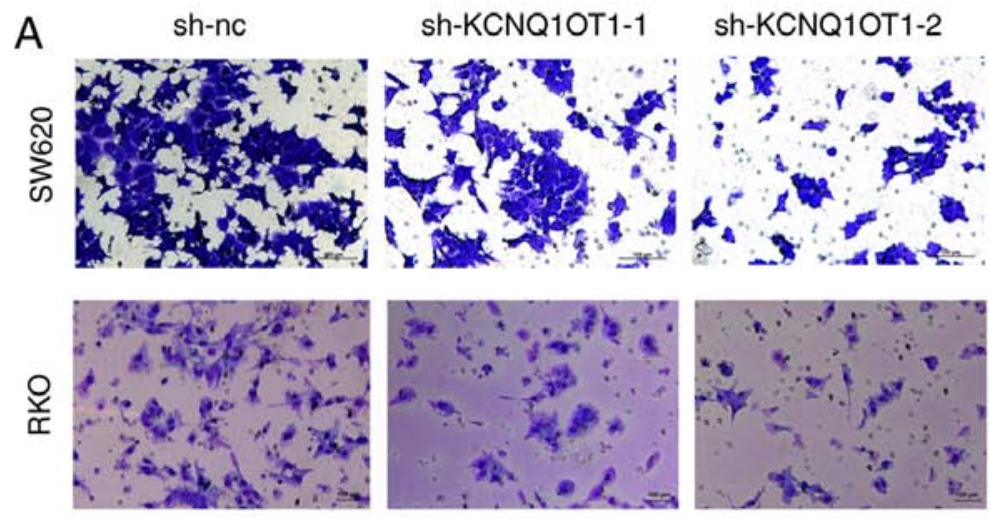

B

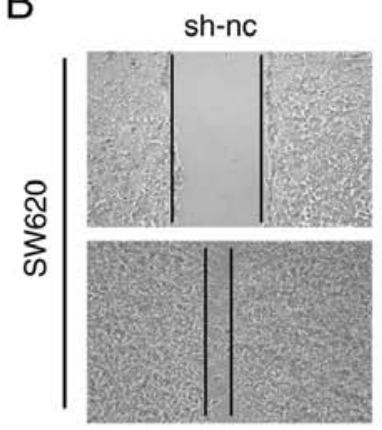

sh-KCNQ1OT1-1

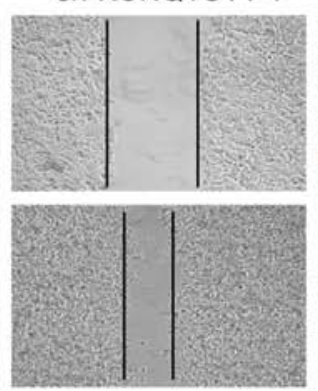

sh-KCNQ1OT1-2
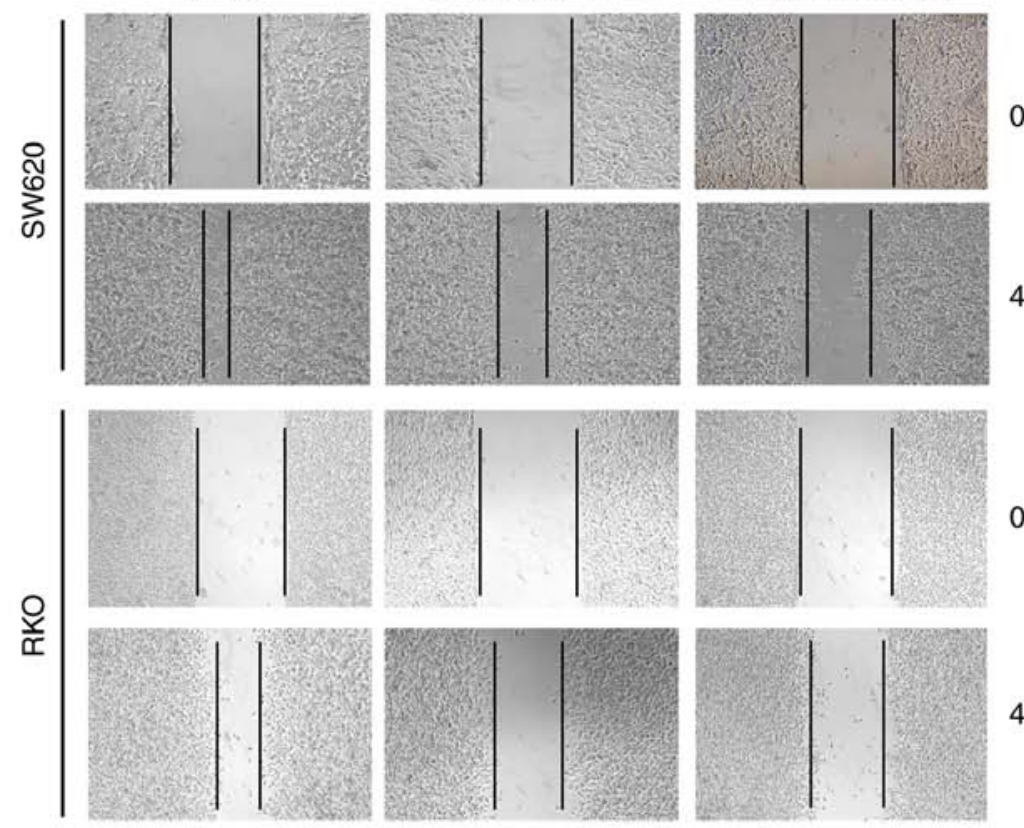

$\mathrm{Oh}$
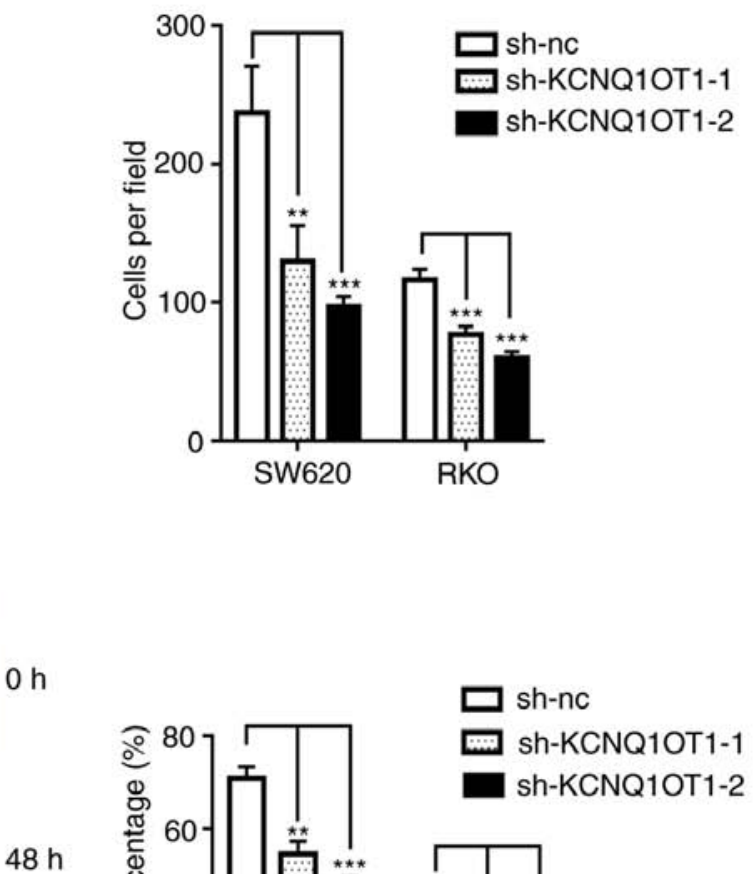

$\mathrm{Oh}$

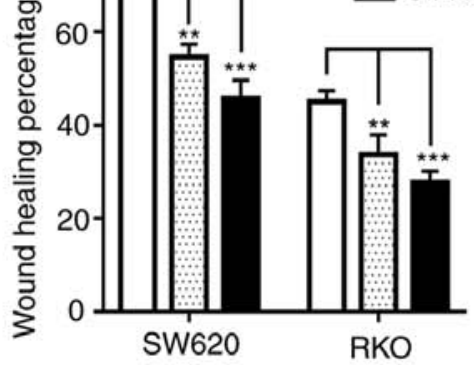

$48 \mathrm{~h}$

Figure 5. KCNQ1OT1 knockdown suppresses colorectal cancer cell migratory and invasive abilities. (A) Number of invading cells in SW620 and RKO cells following KCNQ1OT1 knockdown. (B) Wound healing assay of SW620 and RKO cells following KCNQ1OT1 knockdown. ${ }^{* *} \mathrm{P}<0.01$ and ${ }^{* * *} \mathrm{P}<0.001$. nc, negative control; sh, short hairpin.

KCNQ1OT1 knockdown inactivates the PI3K/AKT pathway, as evidenced by the decreased expression of p-PI3K and p-AKT in SW620 and RKO cell lines (Fig. 6A and B). In addition, following CRC cell treatment with a PI3K inhibitor, the level of p-PI3K and p-AKT was decreased compared with non-treated cells (Fig. 6C and D). The inhibition experiment was successful, confirming that KCNQ1OT1 inhibited the proliferation and invasion of colorectal cancer by inhibiting the PI3K signaling pathway. These data suggest that KCNQ1OT1 knockdown may inhibit PI3K/AKT signaling pathway activation.

\section{Discussion}

Numerous studies have demonstrated that lncRNAs serve crucial roles in malignant tumor progression, including CRC $(5,19)$. For example, Chen et al $(20)$ reported that lncRNA XIST is significantly overexpressed in tumors from patients with CRC and is associated with poor overall survival. Furthermore, it was demonstrated that XIST can regulate CRC progression and metastasis by competing with the micro-RNA miR-200b for the regulation of zinc finger E-box binding homeobox 1 expression (20). In addition, Han et al (21) reported that IncRNA CRNDE could promote chemoresistance and CRC cell proliferation by regulating the expression levels of miR-181a-5p and the activity of the Wnt/ $\beta$-catenin signaling pathway. A previous study reported that $\beta$-catenin could directly target KCNQ1OT1 to promote CRC development (22). However, the role and underlying mechanism of KCNQ1OT1 in CRC remains unclear.

The present study demonstrated that KCNQ1OT1 is highly expressed in CRC tissues compared with normal tissues, and that high KCNQ1OT1 expression in CRC tissues is associated with poor survival according to GEPIA predictions. These results were similar to those observed in $28 \mathrm{CRC}$ tissue samples, in which KCNQ1OT1 was upregulated compared with adjacent normal tissues. Furthermore, KCNQ1OT1 expression was significantly associated with tumor size $(<5 \mathrm{~cm}$ vs. $\geq 5 \mathrm{~cm})$ and clinical stage (limited vs. advanced). These results suggest that high KCNQ1OT1 expression may be 
A

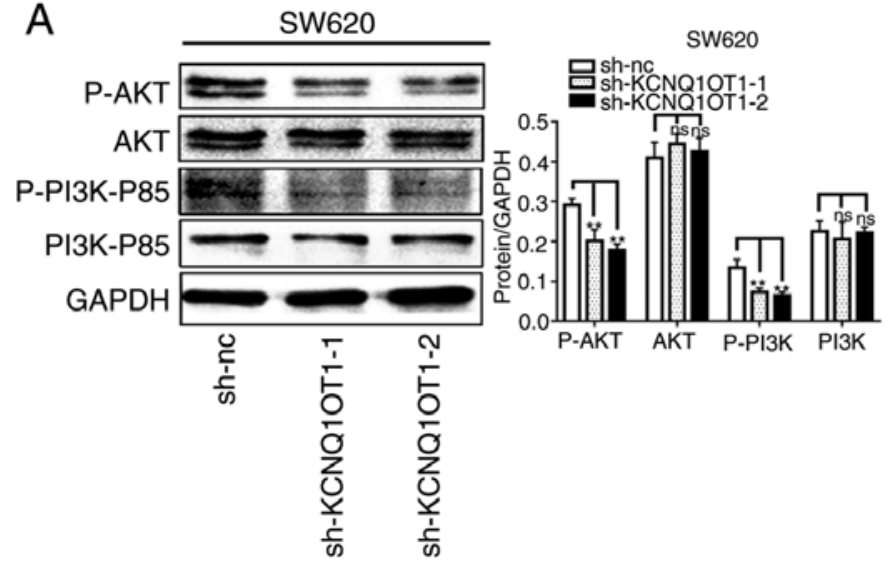

B

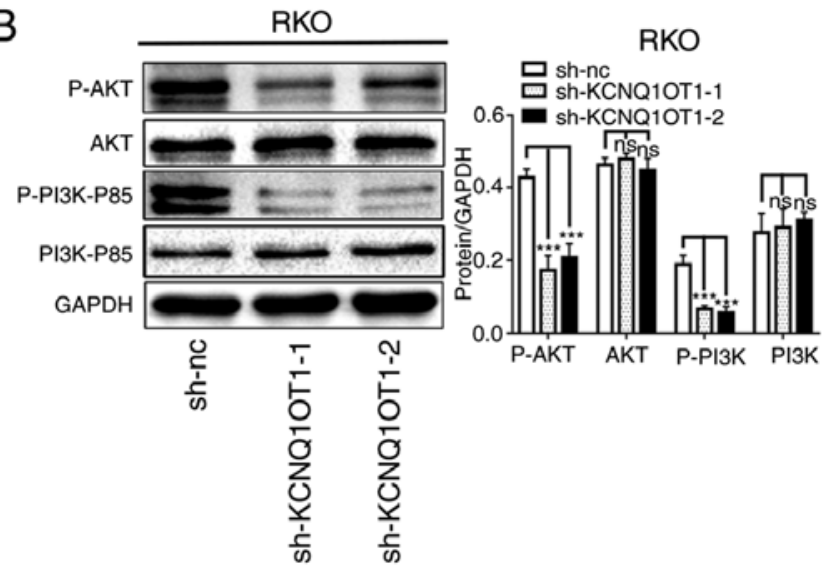

C

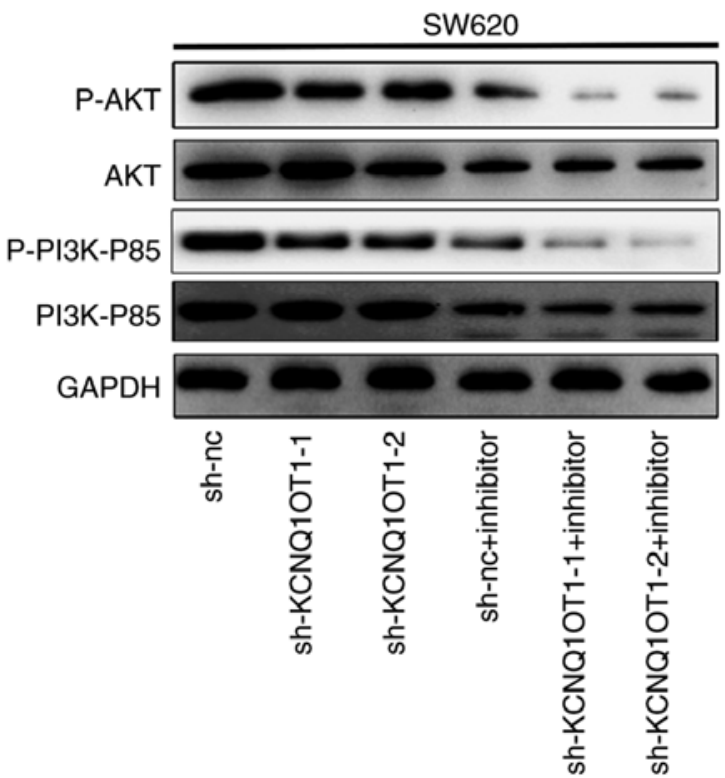

D

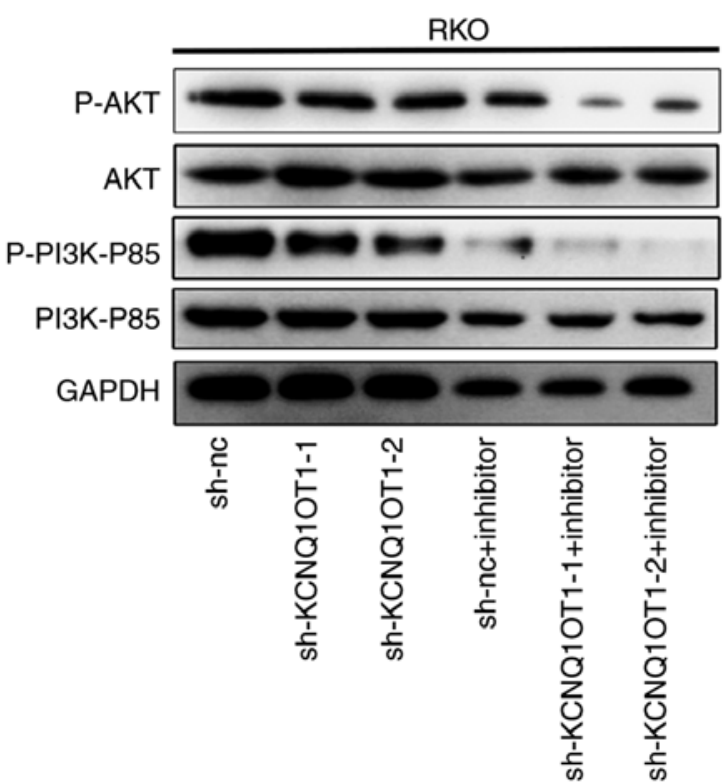

Figure 6. KCNQ1OT1 knockdown inhibits activation of the PI3K/AKT signaling pathway. Relative expression of PI3K, p-PI3K, AKT and p-AKT in (A) SW620 and (B) RKO cells following KCNQ1OT1 knockdown. Relative expression of PI3K, p-PI3K, AKT and p-AKT in (C) SW620 and (D) RKO cells following KCNQ1OT1 knockdown and/or treatment with PI3K inhibitor (10 $\mu \mathrm{M} \mathrm{LY294002} \mathrm{for} 24 \mathrm{~h}){ }^{* *} \mathrm{P}<0.01$ and ${ }^{* * * *} \mathrm{P}<0.001$. nc, negative control; ns, not significant; $\mathrm{p}$, phosphorylated; sh, short hairpin.

associated with the malignant progression of CRC. Previous research demonstrates that KCNQ1OT1 overexpression serves a crucial role in various types of tumor. For example, it was reported that KCNQ1OT1 is upregulated in chemoresistant tongue squamous cell carcinoma tissues compared with chemosensitive tissues, and that high KNCQ1OT1 expression is positively correlated with poor prognosis in patients (8). Another study demonstrated that KCNQ1OT1 is highly expressed and promotes tumor growth and metastasis in cholangiocarcinoma via miR-140-5p/SRY-box transcription factor 4 axis (23). It was demonstrated that KCNQ1OT1 is upregulated in patients with hepatocellular carcinoma (9), breast cancer (10), non-small-cell lung cancer (15) and glioma (24), and that it promotes malignant tumor progression. The results from these studies indicate that KCNQ1OT1 may serve an oncogenic role in various types of cancer.

The present study demonstrated that KCNQ1OT1 knockdown inhibited the proliferation and migratory and invasive abilities of CRC cells, which was consistent with previous studies on cholangiocarcinoma and hepatocellular carcinoma $(9,23)$. Numerous studies have reported that the cell cycle and apoptosis are associated with cancer cell proliferation and tumor growth $(16,25,26)$. Subsequently, KCNQ1OT1 was knocked down in the present study and the cell cycle and apoptosis were evaluated. The results demonstrated that KCNQ1OT1 knockdown promoted apoptosis and cell cycle arrest in CRC cells. These findings indicate that KCNQ1OT1 may regulate CRC cell proliferation, migratory and invasive abilities, cell cycle and apoptosis in order to promote tumor growth and metastasis.

The PI3K/AKT signaling pathway serves an essential role in the growth and metastasis of multiple tumors. For example, lncRNA ABHD11-AS1 promotes tumor progression by regulating PI3K/AKT signaling in papillary thyroid carcinoma (27). In addition, lncRNA SNHG7 facilitates CRC cell proliferation and metastasis via the PI3K/AKT/mTOR signaling pathway, and by regulating GALNT7 expression via miR-34a sponging (28). Following PI3K/AKT pathway inactivation, $\mathrm{G}_{1} / \mathrm{S}$ transition, proliferation and migratory and invasive abilities of cancer cells are inhibited, whereas 
apoptosis is stimulated $(17,28)$. The results from the present study demonstrate that KCNQ1OT1 knockdown decreases the protein expression of p-PI3K and p-AKT. These findings suggest that $\mathrm{CRC}$ cell proliferation and metastasis may be regulated by KCNQ1OT1 via the PI3K/AKT signaling pathway.

In conclusion, the present study demonstrated that KCNQ1OT1 expression was significantly increased in CRC cells and tissues compared with normal cells and tissues. Furthermore, KCNQ1OT1 knockdown inhibited CRC cell proliferation and migratory and invasive ability, promoted CRC cell apoptosis and arrested the cell cycle via PI3K/AKT signal pathway inactivation. These findings indicate that KCNQ1OT1 may be considered as a potential prognostic marker and therapeutic target for CRC. However, the study had several limitations. For example, the effect of KCNQ1OT1 overexpression in CRC cells in vitro or its function in vivo, were not investigated. Future studies will further explore the underlying mechanisms of KCNQ1OT1 in CRC, in particular, its potential role as a competitive endogenous RNA.

\section{Acknowledgements}

Not applicable.

\section{Funding}

The present study was supported by the Natural Science Foundation of Guangdong Province, China (grant no. 2017A030313533).

\section{Availability of data and materials}

The data generated during the study are included in this article.

\section{Authors' contributions}

JY and CC designed the present study. QD and LC conducted the main experiments and drafted the initial manuscript. $\mathrm{KZ}$ conducted some minor experiments in this study. $\mathrm{RH}$, CW and LX analyzed the bioinformatics databases and assessed the expression levels in clinical tissues. ZZ and $X Y$ analyzed the data. All authors read and approved the final manuscript.

\section{Ethics approval and consent to participate}

The present study was approved by the Ethics Committee of Zhujiang Hospital, Southern Medical University (approval no. 2018-PTWK-005). All patients signed informed consent prior to the study.

\section{Patient consent for publication}

Not applicable.

\section{Competing interests}

The authors declare that they have no competing interests.

\section{References}

1. Torre LA, Bray F, Siegel RL, Ferlay J, Lortet-Tieulent J and Jemal A: Global cancer statistics, 2012. CA Cancer J Clin 65: 87-108, 2015

2. Li Y, Liang L, Dai W, Cai G, Xu Y, Li X, Li Q and Cai S: Prognostic impact of programed cell death-1 (PD-1) and PD-ligand 1 (PD-L1) expression in cancer cells and tumor infiltrating lymphocytes in colorectal cancer. Mol Cancer 15: 55, 2016.

3. Markowitz SD and Bertagnolli MM: Molecular origins of cancer: Molecular basis of colorectal cancer. N Engl J Med 361: 2449-2460, 2009.

4. ENCODE Project Consortium: An integrated encyclopedia of DNA elements in the human genome. Nature 489: 57-74, 2012.

5. Esteller M: Non-coding RNAs in human disease. Nat Rev Genet 12: 861-874, 2011.

6. Wang KC and Chang HY: Molecular mechanisms of long noncoding RNAs. Mol Cell 43: 904-914, 2011.

7. Schmitz SU, Grote P and Herrmann BG: Mechanisms of long noncoding RNA function in development and disease. Cell Mol Life Sci 73: 2491-2509, 2016.

8. Zhang S, Ma H, Zhang D, Xie S, Wang W, Li Q, Lin Z and Wang Y: LncRNA KCNQ1OT1 regulates proliferation and cisplatin resistance in tongue cancer via miR-211-5p mediated Ezrin/Fak/Src signaling. Cell Death Dis 9: 742, 2018.

9. Li C, Miao R, Zhang J, Qu K and Liu C: Long non-coding RNA KCNQ1OT1 mediates the growth of hepatocellular carcinoma by functioning as a competing endogenous RNA of miR-504. Int J Oncol 56: 857-858, 2020.

10. Feng W, Wang C, Liang C, Yang H, Chen D, Yu X, Zhao W, Geng D, Li S, Chen Z and Sun M: The dysregulated expression of KCNQ1OT1 and Its interaction with downstream factors miR-145/CCNE2 in breast cancer cells. Cell Physiol Biochem 49: 432-446, 2018.

11. NCCN Clinical Practice Guidelines in Oncology (NCCN Guidelines $^{\circledR}$ )-Colon Cancer v1.2019. Accessed March 15, 2019.

12. Tang Z, Li C, Kang B, Gao G, Li C and Zhang Z: GEPIA: A web server for cancer and normal gene expression profiling and interactive analyses. Nucleic Acids Res 45: W98-W102, 2017.

13. Wang Y, Kuang H, Xue J, Liao L, Yin F and Zhou X: LncRNA AB073614 regulates proliferation and metastasis of colorectal cancer cells via the PI3K/AKT signaling pathway. Biomed Pharmacother 93: 1230-1237, 2017.

14. Livak KJ and Schmittgen TD: Analysis of relative gene expression data using real-time quantitative PCR and the 2(-Delta Delta C(T)) method. Methods 25: 402-408, 2001.

15. Dong Z, Yang P, Qiu X, Liang S, Guan B, Yang H, Li F, Sun L, Liu H, Zou G and Zhao K: KCNQ1OT1 facilitates progression of non-small-cell lung carcinoma via modulating miRNA-27b-3p/HSP90AA1 axis. J Cell Physiol 234: 11304-11314, 2019.

16. Evan GI and Vousden KH: Proliferation, cell cycle and apoptosis in cancer. Nature 411: 342-348, 2001.

17. Huang JL, Cao SW, Ou QS, Yang B, Zheng SH, Tang J, Chen J, Hu YW, Zheng L and Wang Q: The long non-coding RNA PTTG3P promotes cell growth and metastasis via up-regulating PTTG1 and activating PI3K/AKT signaling in hepatocellular carcinoma. Mol Cancer 17: 93, 2018.

18. Lien EC, Dibble CC and Toker A: PI3K signaling in cancer: Beyond AKT. Curr Opin Cell Biol 45: 62-71, 2017.

19. Schmitt AM and Chang HY: Long noncoding RNAs in cancer pathways. Cancer Cell 29: 452-463, 2016.

20. Chen DL, Chen LZ, Lu YX, Zhang DS, Zeng ZL, Pan ZZ, Huang P, Wang FH, Li YH, Ju HQ and Xu RH: Long noncoding RNA XIST expedites metastasis and modulates epithelial-mesenchymal transition in colorectal cancer. Cell Death Dis 8: e3011, 2017.

21. Han P, Li JW, Zhang BM, Lv JC, Li YM, Gu XY, Yu ZW, Jia YH, Bai XF, Li L, et al: The lncRNA CRNDE promotes colorectal cancer cell proliferation and chemoresistance via miR-181a-5p-mediated regulation of $\mathrm{Wnt} / \beta$-catenin signaling. Mol Cancer 16: 9, 2017.

22. Sunamura N, Ohira T, Kataoka M, Inaoka D, Tanabe H, Nakayama Y, Oshimura $M$ and Kugoh $H$ : Regulation of functional KCNQ1OT1 lncRNA by $\beta$-catenin. Sci Rep 6: 20690, 2016.

23. Sun H, Li Y, Kong H, Dai S and Qian H: Dysregulation of KCNQ1OT1 promotes cholangiocarcinoma progression via miR-140-5p/SOX4 axis. Arch Biochem Biophys 658: 7-15, 2018. 
24. Gong W, Zheng J, Liu X, Liu Y, Guo J, Gao Y, Tao W, Chen J, $\mathrm{Li} Z$, Ma J and Xue Y: Knockdown of long non-coding RNA KCNQ1OT1 restrained glioma cells' malignancy by activating miR-370/CCNE2 axis. Front Cell Neurosci 11: 84, 2017.

25. Huang M, Wang H, Hu X and Cao X: lncRNA MALAT1 binds chromatin remodeling subunit BRG1 to epigenetically promote inflammation-related hepatocellular carcinoma progression. Oncoimmunology 8: e1518628, 2018.

26. Wang $Z$ and Qin B: Prognostic and clinicopathological significance of long noncoding RNA CTD-2510F5.4 in gastric cancer. Gastric Cancer 22: 692-704, 2019.

27. Wen J, Wang H, Dong T, Gan P, Fang H, Wu S, Li J, Zhang Y, Du R and Zhu Q: STAT3-induced upregulation of lncRNA ABHD11-AS1 promotes tumour progression in papillary thyroid carcinoma by regulating miR-1301-3p/STAT3 axis and PI3K/AKT signalling pathway. Cell Prolif 52: e12569, 2019.
28. Li Y, Zeng C, Hu J, Pan Y, Shan Y, Liu B and Jia L: Long non-coding RNA-SNHG7 acts as a target of miR-34a to increase GALNT7 level and regulate PI3K/Akt/mTOR pathway in colorectal cancer progression. J Hematol Oncol 11: 89, 2018.

This work is licensed under a Creative Commons Attribution-NonCommercial-NoDerivatives 4.0 International (CC BY-NC-ND 4.0) License. 